\title{
The white dwarf cooling sequence in the old open cluster NGC 188*
}

\author{
G. Andreuzzi ${ }^{1}$, H. B. Richer ${ }^{2}$, M. Limongi ${ }^{1}$, and M. Bolte ${ }^{3}$ \\ 1 INAF-Astronomical Observatory of Rome, via di Frascati I-33, 00040 Monteporzio Catone, Roma, Italy \\ 2 Department of Physics and Astronomy, University of British Columbia, Vancouver, BC V6T 1Z1, Canada \\ ${ }^{3}$ University of California, UCO/Lick Observatory, Santa Cruz, CA 95064, USA
}

Received 17 January 2002 / Accepted 6 June 2002

\begin{abstract}
We develop the white dwarf luminosity function (LF) of the old open cluster NGC 188 in order to determine a lower limit to the age of the cluster by using the faint end of the cooling sequence.

To produce an extensive sequence of the cooling white dwarfs we imaged four contiguous HST-WFPC2 fields in the center of the cluster in the F555W and F814W filters. After imposing selection criteria on the detected objects we found a white dwarf cooling sequence (down to $V \simeq 26.5$ ) including 28 candidate white dwarfs in the cluster. The exposures are not deep enough to reach the end of this sequence, but the results of our analysis allow us to establish a lower limit to the age of the cluster independently of the isochrone fit to the cluster turnoff.

The most ancient white dwarfs found are $\simeq 4$ Gyr old, an age that is set solely by the photometric limit of our data. Classical methods provide an estimate of $\simeq 7$ Gyr (Sarajedini et al. 1999).
\end{abstract}

Key words. open clusters and associations: individual: NGC 188 - stars: white dwarfs - stars: luminosity function

\section{Introduction}

Stellar evolution theory predicts that all single stars having a Main Sequence (MS) mass lower than $\simeq 8 M_{\odot}$ end their lives as white dwarfs (WDs). In a star cluster, the white dwarf population carries key information for addressing a number of astrophysical questions. In particular, since the white dwarfs cool at a predictable rate, their LF can be used to constrain the cluster age. This age estimate can provide an independent test of the more traditional age determinations based on models and observations of the main-sequence turnoff (MSTO).

The white dwarf cooling sequence in the color-magnitude diagram, in combination with models can be used to estimate the initial mass - final mass relationship between the stellar progenitors and the white dwarfs over the range of progenitor mass from near $8 M_{\odot}$ to the current-day turnoff mass of a cluster. This allows a determination of the amount of mass lost from stars during their evolution through stellar winds and planetary nebula ejection.

Ideally, to employ white dwarfs as age estimators, we need to observe a large enough sample in any cluster to define the WDLF sufficiently well to pinpoint the luminosity of the

Send offprint requests to: G. Andreuzzi,

e-mail: gloria@coma.mporzio.astro.it

* Tables with the $x, y$ coordinates, $V$ and $I$ magnitudes, are only available in electronic form at the CDS via anonymous ftp to cdsarc.u-strasbg.fr (130.79.128.5) or via

http://cdsweb.u-strasbg.fr/cgi-bin/qcat?]/A+A/390/961 turndown with a precision equivalent to a Gyr of cooling time. With smaller samples, it is still possible to make some progress on the age questions. For example, even a single good candidate WD in a cluster at magnitudes fainter than the faintest WDs predicted from the MSTO age and cooling theory can bring into question the vailidity of the WD or MSTO-based ages.

Clearly the most interesting case for testing MSTO-based ages is for the globular clusters. However, because of the combination of large age and large distance for even the nearest of the Galactic globulars, it is not trivial to reach the end of the cooling sequence (e.g. Richer et al. 1995, 1997, 2002; Hansen et al. 2002). Old open clusters provide an interesting alternative for WD-cooling age studies. There are several populous clusters which are both nearer and younger than the nearest globulars and for which the expected WDLF turndown is 3 mag or more brighter. For open clusters older than $\sim 4$ Gyr, the mainsequence turnoff stars are very similar in structure to globular cluster turnoff stars, most importantly in that they have radiative cores, and comparison of nuclear and cooling ages for these old open clusters are very relevant the issue of globular cluster MSTO age tests. A significant difficulty with open clusters is that even the most populous are sparse compared to most globular clusters. This leads to the problem that the lowest luminosity WDs are typically dramatically outnumbered by faint blue galaxies in the cluster fields. Attempts to statistically derive a WDLF by subtracting counts from nearby control fields are compromised by small errors in control-field 
counts and cosmic dispersion in faint blue galaxy surface density. Very accurate star-galaxy separation is therefore crucial for open cluster WD studies. Given the compact size of many faint blue galaxies, Hubble Space Telescope (HST) imaging gives a tremendous advantage. Ultimately another powerful technique for isolating cluster-member WDs from the compact blue galaxy and field WD populations may be measuring proper motions.

NGC 188 was considered for a long time to be the oldest observable open cluster in the Galaxy. Almost 40 years ago Sandage gave an estimate for the age of this cluster of $10 \mathrm{Gyr}$ (Sandage 1962; see also Eggen \& Sandage 1969). Recent papers revise the early age estimates downward, and more modern values give an age near 7 Gyr (see Sarajedini et al. 1999). Other open clusters have now been found to be older than NGC 188 (NGC6791 and Berkeley 17 with ages of 8 and 12 Gyr respectively, see Phelps et al. 1994) however, NGC 188 is still one of the most studied since it is both nearer and less obscured than some of the other old open clusters.

Sections 2 and 3 present the data, the reduction procedures and the color magnitude diagrams. The resulting LFs together with final results are presented and discussed in Sects. 4 and 5.

\section{Observations and data reduction}

Deep HST WFPC2 images in F555W and F814W were obtained around the centre of NGC 188 in the Cycle 7 program GO 7371. The observations consisted of four fields, f1, f2, f3 and 44 located as shown in Table 1, in such a way that WF4 of the field $\mathrm{f} 4$ overlaps WF2 of the field $\mathrm{f} 1$ and WF4 of the field f3 overlaps WF2 of the field f2. Table 1 also lists the corresponding exposure times together with the number of observed frames for each filter.

Table 1. Journal of observations.

\begin{tabular}{c|cccc}
\hline \hline & $\alpha$ & $\delta$ & F555W & F814W \\
\hline f1 & $0^{\mathrm{h}} 48^{\mathrm{m}} 00^{\mathrm{s}}$ & $85^{\circ} 13^{\prime} 30^{\prime \prime}$ & $4 \times 1400$ & $4 \times 1400$ \\
f2 & $0^{\mathrm{h}} 48^{\mathrm{m}} 00^{\mathrm{s}}$ & $85^{\circ} 16^{\prime} 00^{\prime \prime}$ & $4 \times 1400$ & $4 \times 1400$ \\
f3 & $0^{\mathrm{h}} 46^{\mathrm{m}} 00^{\mathrm{s}}$ & $85^{\circ} 16^{\prime} 00^{\prime \prime}$ & $5 \times 1400$ & $4 \times 1400$ \\
$\mathrm{f} 4$ & $0^{\mathrm{h}} 46^{\mathrm{m}} 00^{\mathrm{s}}$ & $85^{\circ} 13^{\prime} 30^{\prime \prime}$ & $3 \times 1300$ & $3 \times 1400$ \\
\hline
\end{tabular}

Corrections to the raw data for bias, dark and flat-fielding were performed using the standard HST pipeline. Before reduction, we masked bad pixels and corrected the images for vignetting by using a mask and a vignetting mask respectively as built for HST data (supplied by P. Stetson). We also multiplied our science exposures by a pixel-area map to correct for geometrical distortion and to restore the integrity of the flux measurements. Photometry was measured using ALLFRAME (Stetson-version 3, 1997) with a quadratically varying point spread function (PSF).

The images did not have enough bright unsaturated stars to create proper PSFs, so PSFs for each chip were constructed using WFPC2 images of the globular cluster $\omega$ Cen images (P. B. Stetson 1997, private communication). Since there were virtually no stars in the PC frames, these were not included in the

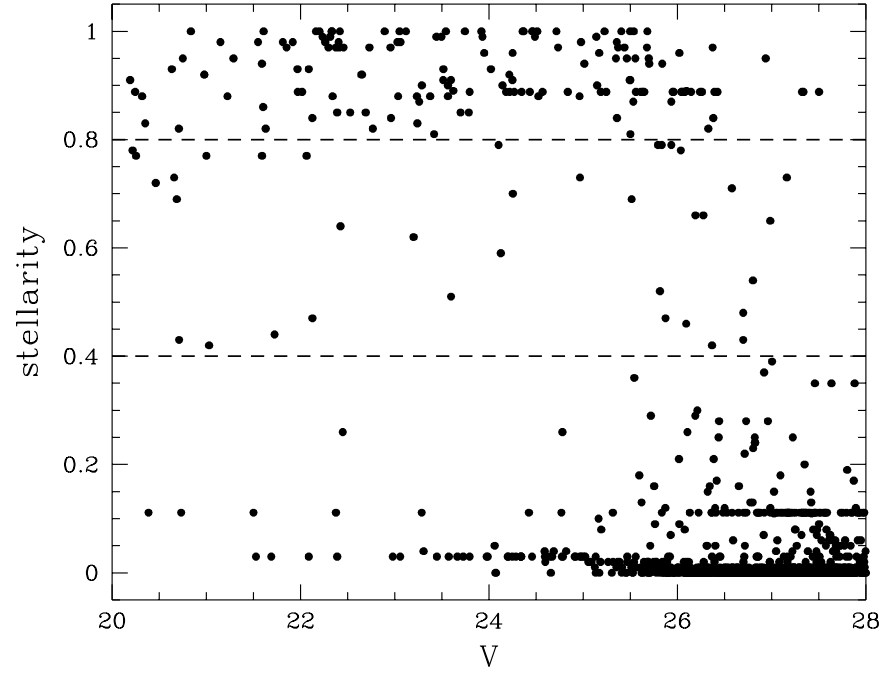

Fig. 1. SExtractor stellarity index vs. the magnitude $V$ for objects in our sample. The horizontal dashed lines at $c=0.4$ and $c=0.8$ show the thresholds we used to distinguish between real stars and other objects (i.e. cosmic rays, image defects, galaxies).

reduction and analysis. The data reduction process was completed by doing the charge transfer efficiency (CTE) correction on the data-set by using an algorithm supplied by P. B. Stetson (priv. comm.).

Finally the instrumental magnitudes were transformed into the standard Johnson system following Holtzman et al. (1995).

To separate stars from other objects at faint magnitudes below $V \simeq 24.5$, we applied SExtractor to the images (Bertin et al. 1996). This program uses a neural network to distinguish between faint point sources from extended objects. It assignes a stellarity index $c$ to the objects, ranging from 0 (galaxies) to 1 (stars). Figure 1 shows the results of the SExtractor morphological classification versus the $V$ magnitude for all the objects in our sample. Looking at the figure there is a clear separation between the objects with stellarity index around 1 and those with stellarity index around 0 and there is very little in between.

Careful examination of each object by eye showed that virtually all the objects for which $0 \leq c<0.4$ are classifiables as galaxies, hot and warm pixels, ghost images or part of diffraction spikes; all the objects for which $0.4 \leq c<0.8$ are dubious objects; all the objects for which $c \geq 0.8$ may be considered stars.

Using these criteria, we established a catalog of 157 stars in all the chips and all the fields studied selected from a total number of 1342 objects.

\section{Data analysis}

\subsection{The colour-magnitude diagram and the WD cooling sequence}

The $V, V-I$ CMD for all stars (as indicated from the stellarity index) measured in the four fields is shown in Fig. 2.

There is a fairly well-defined main sequence extending almost 6 mag from $V \simeq 19$ down to $V \simeq 25$. The solid line 


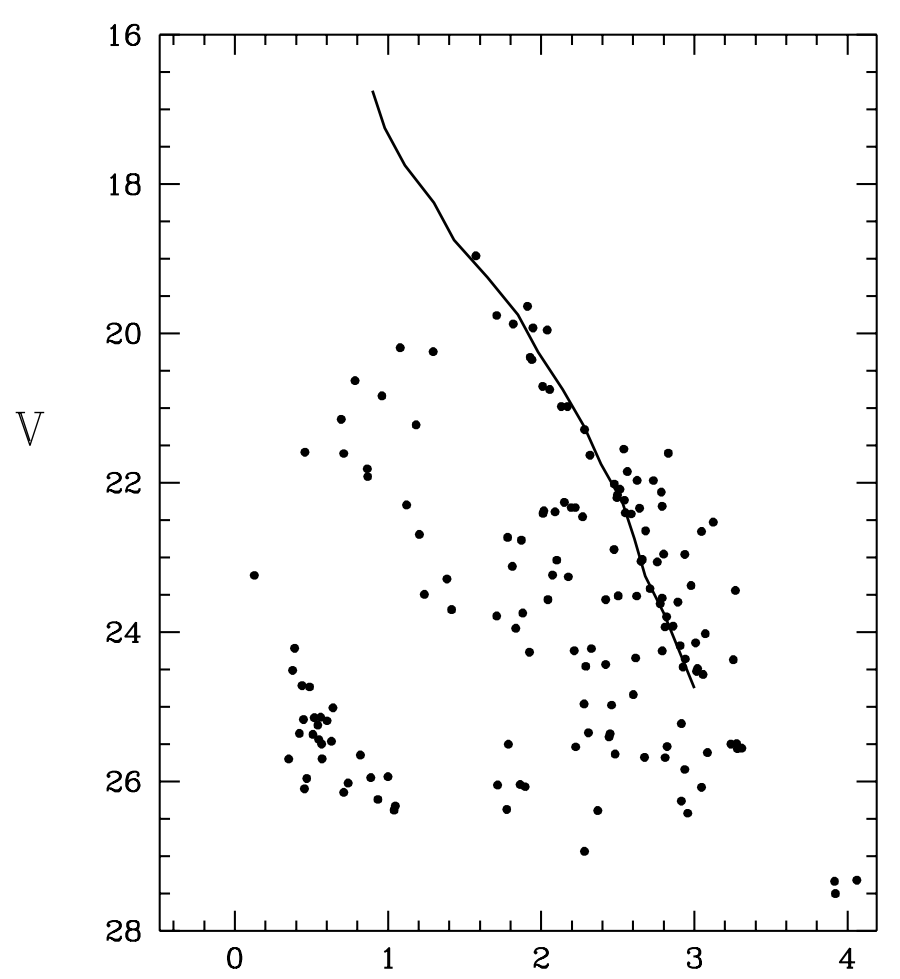

$\mathrm{V}-\mathrm{I}$

Fig. 2. $V-I$ colour-magnitude diagram for the objects in the four observed fields. The solid line overlayed on the data is the ridge line obtained by using the data sample from von Hippel et al. (1998).

overlaid on our data is the ridge line obtained by using a sample of ground-based data (von Hippel et al. 1998).

In the lower left of the CMD there are 28 blue objects ranging from $V \simeq 23$ with $V-I \simeq 0$ to $V \simeq 26.5$ with $V-I \simeq 1$. An enlargement of this region of the CMD is shown in Fig. 3; for each star we also plotted the associated photometric error as computed by ALLFRAME.

Note that in this figure all objects appear to be stars but, for now, we cannot discriminate between those belonging to the cluster and those belonging to the Galactic field. We expect a contamination of $\simeq 8$ Galactic white dwarfs at the magnitude and colour of interest for our analysis (see Mendez \& Minniti 2000). The correction for the field contamination will be addressed in the following sections.

We can use the CMD to estimate the expected number of WDs in the cluster, starting from the number of the MS stars. To carry out this consistency check, we have found the slope of the mass function of the cluster by using the number of MS stars in the $V$ magnitude range 20-24, obtaining a value of 1.72 (Salpeter is 2.35). We then estimated the mass of the stars at the TO under the hypothesis that the cluster age is 4-5 Gyr, obtaining respectively $\sim 1.23 M_{\odot}$ and $1.15 M_{\odot}$. By looking at the coolest and faintest WDs inside the range in magnitude $V$ (23-26), and adopting a WD mass of $0.6 M_{\odot}$, we obtained only 3 and 4 expected WDs in the cluster (respectively for 4 and $5 \mathrm{Gyr}$ ), a very small number if compared with the observed number in the same range in magnitude (23 WDs).

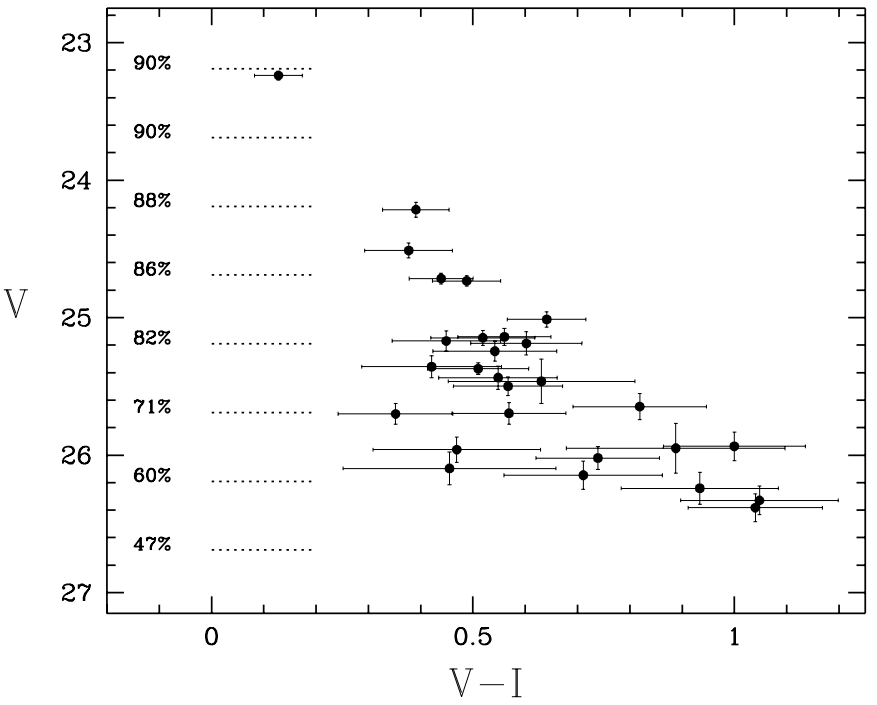

Fig. 3. $V-I$ colour-magnitude diagram for all possible white dwarf candidates in our sample. Objects were selected based on their stellarity index $(c \geq 0.8)$ and, at this stage, there is no correction for field contamination. For each object we have also plotted the associated error based on the frame-to-frame scatter in the measurements. Dotted lines (and the percentages) on the left indicate the mean completeness for each bin at the respective $V$ magnitude.

We emphasize that this result is a consequence of the assumptions on the poorly determined mass function slope. For example, for a slope of 1.05 , this number changes to 13 or 22 for cluster ages of 4 or $5 \mathrm{Gyr}$ respectively. If we then are to believe that most of the objects in the observed cooling sequence are in fact WDs, we need to assume that our sample is somehow deficient in MS stars. This is not unexpected for NGC 188 because, as suggested by many authors (Sarajedini et al. 1999; von Hippel et al. 1998), dynamical processes and mass segregation are important in the history of this cluster which has the rather short relaxation time of $t_{\mathrm{rh}}=6.4 \times 10^{7} \mathrm{yr}$ (McClure et al. 1977).

\subsection{Completeness correction}

Figure 3 also indicates the completeness in the counts computed in bins of $0.5 \mathrm{mag}$ in $V$. Incompleteness corrections were determined by carrying out artificial star tests on both sets of the $V$ and $I$ frames, so as to be able to estimate the overall completeness of our final colour magnitude diagram in the region of the candidate white dwarfs. To accomplish this we first applied artificial star tests to the $I$ images. Artificial stars were added to random positions in the images over a range of 3 mag in the region of the CMD of interest for the candidate white dwarfs. We created 5 artificial images for each original image each with 100 artificial stars. We emphasize that crowding was not a problem in our images.

We then added an equal number of stars at the same position of the artificial $I$ stars to the $V$-band frames and with a magnitude such that they would fall along the white dwarf cooling sequence. All the $V$ and $I$ frames with artificial stars 
were then subjected to the same analysis adopted for the original frames.

An artificial star was considered recovered if $\delta X, \delta Y \leq$ 1.5 pixel and $\delta \mathrm{mag} \leq 0.3 \mathrm{mag}$. The ratio $N_{\text {rec }} / N_{\text {sim }}=\Phi$, taken in $0.5 \mathrm{mag}$ bins gives the completeness in that bin for the image considered, where $N_{\text {rec }}$ is the number of the recovered stars and $N_{\text {sim }}$ is the number of simulated stars.

\subsection{Accounting for the field white dwarfs}

In addition to correction for photometric incompleteness it is necessary to account for the contamination of the field stars. The majority of the field star contamination is due to mainsequence stars from the Galactic disk together with a few mainsequence stars from the thick disk and the halo. The majority of these objects have much redder colors than our WD candidates in NGC 188.

We have estimated the correction to our WD candidate counts, by assuming that all resolved galaxies have already been eliminated from our data-sample. We then used the number of unresolved blue objects identified by Mendez \& Minniti (2000) in the Hubble Deep Field North in an area $\simeq 4$ times smaller than that covered by our fields.

Table 2 lists the counts for 0.5 mag-wide bins in $V$, the completeness factor and the counts corrected for this factor. The last two columns report the number of unresolved blue objects for each bin and our counts after the correction for completeness and for the contribution of the field.

The absolute $V$ magnitudes of Col. 2 have been obtained adopting $(m-M)_{V}=11.44, E_{B-V}=0.09$ (Sarajedini et al. 1999).

Table 2. Luminosity function for the candidate white dwarfs in our sample in the $V$ band in 0.5 mag bins. $N$ is the actual number of stars observed, $\Phi$ is the completeness correction factor in each bin, $N_{\mathrm{c}}$ is the number of stars after the corrections for incompleteness have been applied, $N_{\text {field }}$ is the number of unresolved blue objects as found in Mendez \& Minniti (2000) for the magnitude and the colour of our sample, $N_{\mathrm{s}}$ is the number of stars after the incompleteness correction and the field star subtraction, i.e. the cluster white dwarf luminosity function.

\begin{tabular}{ll|lllcl}
\hline \hline$V$ & $M_{V}$ & $N$ & $\Phi$ & $N_{\mathrm{c}}$ & $N_{\text {field }}$ & $N_{\mathrm{s}}$ \\
\hline 23.19 & 11.75 & 1 & 0.9 & 1.11 & 0 & 1.11 \\
23.69 & 12.25 & 0 & 0.9 & 0. & 0 & 0. \\
24.19 & 12.75 & 1 & 0.88 & 1.14 & 0 & 1.14 \\
24.69 & 13.25 & 3 & 0.86 & 3.49 & 0 & 3.49 \\
25.19 & 13.75 & 9 & 0.82 & 10.97 & 0 & 10.97 \\
25.69 & 14.25 & 6 & 0.71 & 8.45 & 4 & 4.45 \\
26.19 & 14.75 & 8 & 0.60 & 13.33 & 4 & 9.33 \\
\hline
\end{tabular}

\section{Discussion}

To estimate the WD cooling age for NGC 188, our first approach is to compare the observed WD CMD with the theoretical WD isochrones corresponding to different cluster ages.
Figure 4 shows the theoretical WD isochrones corresponding to cluster ages of 1, 2, 3, 4, 5, 6 and 7 Gyr (solid lines), superimposed on the observed NGC 188 WD cooling sequence. In the figure we also plot the theoretical cooling sequences for 0.5, 0.7 and $0.9 M_{\odot}$ hydrogen-rich WD (dotted lines) to indicate the mass range of the WDs in the cluster. In particular most of the WDs cover the mass range included in our models while a small fraction seem to have a mass larger than $0.9 M_{\odot}$. Isochrones and theoretical cooling sequences have been developed using the WD cooling models of Hansen $(1998,1999)$ as described in Richer et al. (2000) and have been transformed to apparent magnitudes and colors appropriate for NGC 188 adopting $E(V-I)=1.21 \cdot E(B-V)$ (based on Eqs. (3a) and (3b) of Cardelli et al. 1989 applied to the F814W filter) and $(m-M)_{V}=11.44$.

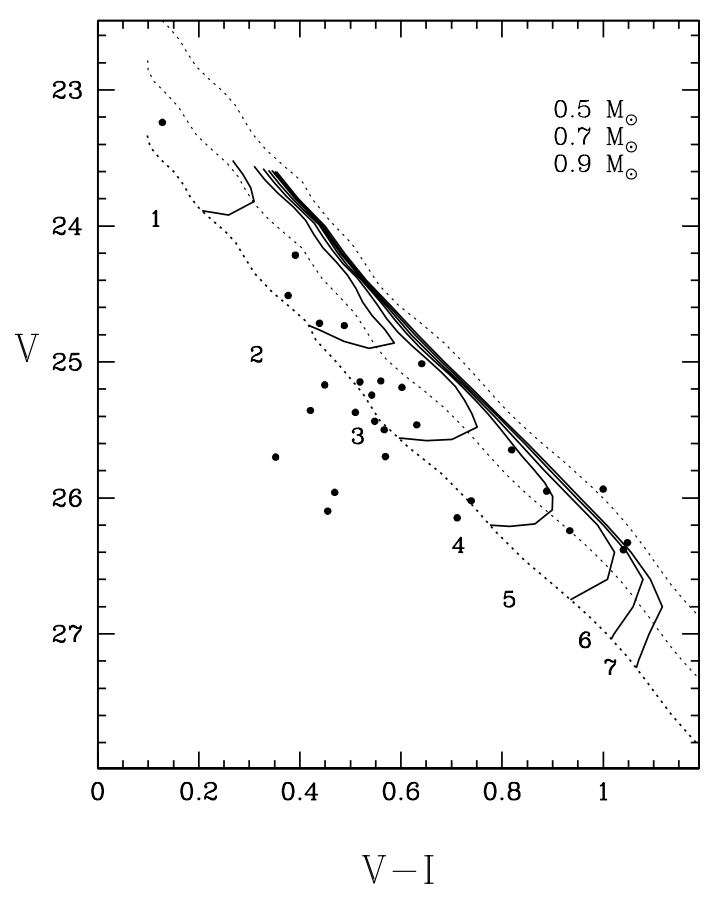

Fig. 4. Comparison of our NGC 188 cooling sequence with theoretical isochrones (solid lines) and hydrogen-rich WD cooling sequences (dashed lines) obtained by using models from Hansen $(1998,1999)$.

Since the population of WDs accumulate at lower luminosities as the age of the cluster increases, we can fix a limit to the age of the cluster by simply looking at the location of the WDs with respect to the theoretical isochrones. Figure 4 seems to show that for NGC 188 this limit is $\sim 6-7$ Gyr. However this method should be used with caution. In fact, when we compare the observed WD CMD and the theoretical isochrones we do not take into account two important corrections that must be applied to the data before carrying out any quantitative conclusion, i.e., the correction for incompleteness and the subtraction of the field contamination. These corrections may be negligible when the sample is composed of many stars but they become very important when we are dealing with such few objects.

The second approach we can follow to derive an estimate of the age of NGC 188 is that of studying the observed WDLF. 


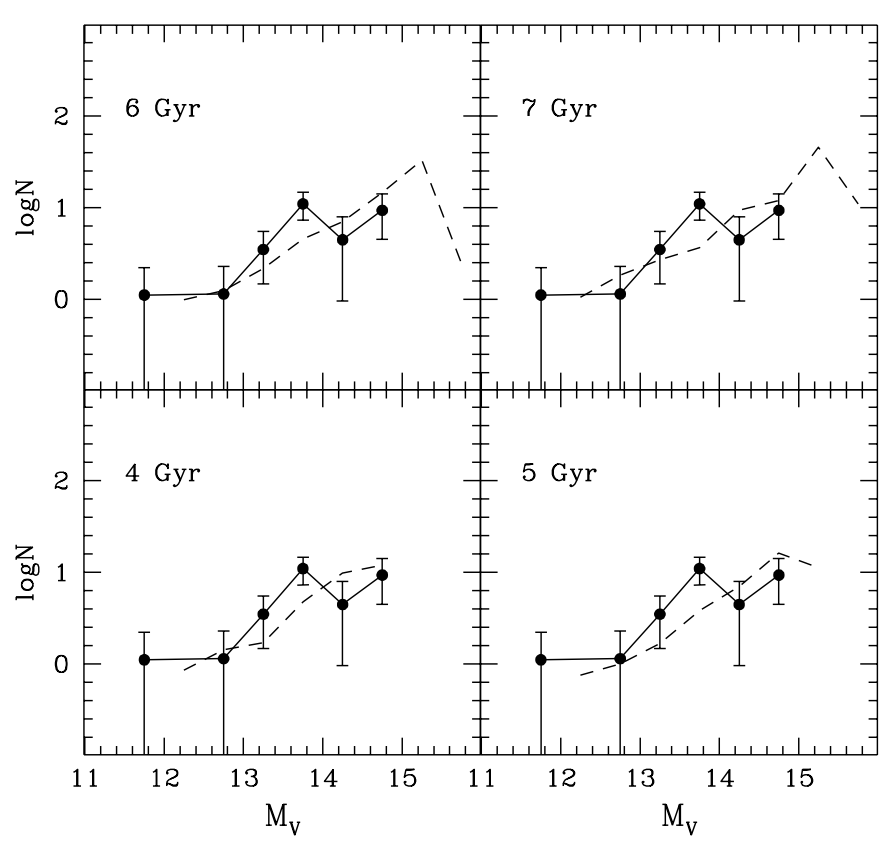

Fig. 5. Differential NGC 188 white dwarf luminosity function (solid line), compared with synthetic luminosity functions of ages 4, 5, 6 and $7 \mathrm{Gyr}$ (dashed lines).

As already mentioned above, the population of WDs tend to accumulate at lower luminosities as the age of the cluster increases. Hence the corresponding white dwarf LF will show a peak whose location in magnitude is a function of the cluster age. In particular, the peak of the white dwarf LF moves towards fainter luminosities as the cluster age increases (see Richer et al. 2000).

Figure 5 (solid line) shows the differential observed WDLF after corrections for incompleteness and field contamination have been applied (see Table 2). In the figure error bars reflect the total error associated with each bin and include both the counting uncertainty and the uncertainty due to the correction for the incompleteness:

$\sigma^{2} \approx \frac{N}{\Phi^{2}}+\frac{(1-\Phi) N^{2}}{N_{\text {sim }} \Phi^{3}}$

where $N$ is the number of observed WDs; $\Phi$ is the incompleteness factor and $N_{\text {sim }}$ is the number of simulated stars.

The uncertainty in the number of objects for each bin has been estimated assuming that the counting uncertainies are derived from a Poisson distribution, and that the uncertainties in determining $\Phi$ are derived from a binomial distribution as shown in Bolte (1989).

$\sigma_{N}^{2}=N$

$\sigma_{\Phi}^{2} \approx \frac{\Phi(1-\Phi)}{N_{\text {sim }}}$

Dashed lines in the four panels of the figure refer to 4 synthetic LFs corresponding to 4, 5, 6 and 7 Gyr respectively. They have been computed by adopting a Salpeter initial-mass function (Salpeter 1954, $n(m) \propto m^{-\alpha} ; \alpha=2.35$ ) as described in
Richer et al. (2000) and have been normalized to the total number of WDs brighter than 14.75, which is the magnitude of the faintest observed WD in the observed LF.

Figure 5 shows that the peak of the theoretical LF is located at magnitudes fainter than the last observed data point $\left(M_{V}=\right.$ 14.75) only in the 6 and 7 Gyr cases. Since it is unlikely that our data reach the true end of the cooling sequence, we suggest this is an indication that this cluster is at least as old as 5 Gyr.

\section{Conclusions}

Although we have identified a clear population of objects in NGC 188 with the photometric properties expected of cluster WDs, it is apparent from Fig. 5 that we have not reached the turnover in the WDLF. The comparison with the LF models suggests that we can place a lower limit of 5 Gyr on the age of the cluster.

These observations could be improved significantly with a modest effort, with HST and the ACS. A fainter photometric limit together with an expanded field coverage would allow a large sample of WDs to be identified in the NGC 188 field. NGC 188 stars have a proper motion of 2.3 milli-arcsec with respect to the field and with a followup program, a subsample of bona-fide cluster WDs could be identified on the basis of their proper motions.

Acknowledgements. We warmly thank P. B. Stetson for kindly providing us images and files for data reduction. We also warmly thank P. B. Stetson, G. Marconi and A. Chieffi, for useful discussions, comments and suggestions. Gloria Andreuzzi gratefully acknowledges the hospitality of H. Richer and the University of British Columbia. This work has been supported by MURST/Cofin1999 under the project: "Effect of the dynamics on the canonical and exotic stellar distributions inside galactic globular clusters".

\section{References}

Anthony-Twarog, B. J. 1981, Ph.D. Thesis, Yale University

Bertin, E., \& Arnouts, S. 1996, A\&AS, 117, 393

Bolte, M. 1989, ApJ, 341, 168

Cardelli, J. A., Clayton, G. C., \& Mathis, J. S. 1989, ApJ, 345, 245

Eggen, O. J., \& Sandage, A. R. 1969, ApJ, 158, 669

Hansen, B. M. S., \& Phinney, E. S. 1998, MNRAS, 294, 557

Hansen, B. M. S. 1999, ApJ, 520, 680

Hansen, B. M. S., Brewer, J., Fahlman, G. G., et al. 2002 [astro-ph/0205087]

Holtzman, J. A., Burrows, C. J., Casertano, S., et al. 1995, PASP, 107, 1065

McClure, R. D., \& Twarog, B. A. 1977, AJ, 214, 111

Mendez, R. A., \& Minniti, D. 2000, ApJ, 529, 911

Phelps, R., Janes, K. A., \& Montgomery, K. A. 1994, AJ, 107, 1079

Richer, H. B., Fahlman, G. G., Ibata, R. A., et al. 1995, ApJ, 451L, 17

Richer, H. B., Fahlman, G. G., Ibata, R. A., et al. 1997, ApJ, 484, 741

Richer, H. B., Hansen, B., Limongi, M., et al. 2000, ApJ, 529, 318

Richer, H. B., Brewer, J., Fahlman, G. G., et al. 2002 [astro-ph/0205086]

Salpeter, E. E. 1954, ApJ, 121, 161

Sandage, A. 1962, ApJ, 135, 333

Sarajedini, A., von Hippel, T., Kozhurina-Platais, V., \& Demarque, P. 1999, AJ, 118, 2894

von Hippel, T., \& Sarajedini, A. 1998, AJ, 116, 1789

von Hippel, T., \& Gilmore, G. 2000, AJ, 120,1384 